University of Nebraska - Lincoln

DigitalCommons@University of Nebraska - Lincoln

Faculty Publications from the Harold W. Manter Laboratory of Parasitology

6-1992

\title{
The Evolutionary Origin of Plasmodium falciparum
}

Daniel R. Brooks

University of Toronto,dnlbrooks@gmail.com

Deborah A. McLennan

University of Toronto

Follow this and additional works at: https://digitalcommons.unl.edu/parasitologyfacpubs

Part of the Parasitology Commons

Brooks, Daniel R. and McLennan, Deborah A., "The Evolutionary Origin of Plasmodium falciparum" (1992). Faculty Publications from the Harold W. Manter Laboratory of Parasitology. 248.

https://digitalcommons.unl.edu/parasitologyfacpubs/248

This Article is brought to you for free and open access by the Parasitology, Harold W. Manter Laboratory of at DigitalCommons@University of Nebraska - Lincoln. It has been accepted for inclusion in Faculty Publications from the Harold W. Manter Laboratory of Parasitology by an authorized administrator of DigitalCommons@University of Nebraska - Lincoln. 


\section{CRITICAL COMMENTS}

J. Parasitol., 78(3), 1992, p. 564-566

(C) American Society of Parasitologists 1992

\section{The Evolutionary Origin of Plasmodium falciparum}

Among species of Plasmodium, $P$. falciparum is an enigma. It is unusually pathogenic and is not characterized by the relapsing episodes characteristic of other species. Its primary vector is a species of mosquito that is highly anthropophilic (Anopheles gambiae), and which shows evidence of rapid genetic change apparently coincident with the rise of human agriculture. Plasmodium falciparum has no alternative vertebrate reservoir hosts, and if the number of human infections drops below a threshold level, the species is unable to maintain itself. All of these observations suggested a novel hypothesis to Boyd (1949, cited in Waters et al. [1991]), who proposed that $P$. falciparum is a relatively new species that originated when the advent of agriculture brought humans and certain types of mosquitoes into contact on a regular basis.

Building upon Boyd's hypothesis, Waters et al. (1991) investigated the evolutionary origins of $P$. falciparum in more detail. They utilized fragments of asexually expressed small-subunit ribosomal RNA converted to estimates of genetic distances to generate phylogenetic trees for several species of Plasmodium infecting rodents, primates (including humans), and birds. They concluded that Boyd's hypothesis about the recent origin of P. falciparum was upheld by the position of that parasite on the phylogenetic tree. They further postulated that $P$. falciparum originated via a host switch from birds to humans because it formed a monophyletic group with the 2 species of avian Plasmodium examined. This would require 1 of 2 events: either a species of mosquito that was regularly a vector for some avian-inhabiting Plasmodium species began feeding on humans as well, or a species of mosquito that regularly fed on humans began feeding on birds as well. The high degree of pathogenicity and unusual biology of $P$. falciparum is thus explained by its being a relatively young species and by its origin via a host switch, because it often is assumed that pathogenicity and novel biologies are proportional to the age of an association between a host and a parasite.

Monophyly by itself, however, does not tell us whether the ancestor of $P$. falciparum switched from primates to birds or from birds to primates (Fig. 1). To choose between these 2 alternatives, it is necessary to examine the host relationships of other members of Plasmodi$u m$ and use phylogenetic optimization procedures to infer the direction of host switching. Fortunately, these data were provided by Waters et al. (1991). Phylogenetic optimization (see Brooks and McLennan [1991] and Wiley et al. [1991] and references therein) of host types onto their 2 trees unambiguously supports the conclusion that Plasmodium species infecting birds are derived from a group of Plasmodium species inhabiting primates, specifically human primates (Fig. 2).

Waters et al. (1991) addressed 2 questions. First, from where did $P$. falciparum come? According to the host data and phylogenetic relationships presented in their paper, the ancestor of $P$. falciparum is the sister species of Plasmodium malariae. Its antecedents are thus strictly "human"; i.e., the parasite speciated in association with the ancestral host type. Second, why is $P$. falciparum so virulent? The phylogenetic trees presented by Waters et al. (1991) are consistent with Boyd's hypothesis that $P$. falciparum is the most recently derived species of Plasmodium inhabiting humans. Although the trees do not support their hypothesis that the evolutionary origin of $P$. falciparum is associated with a host switch, this does not preclude a role for the appearance of agriculturally based communities on the parasite's subsequent evolutionary trajectory. The origin of $P$. falciparum may be explained by an interaction between its recent age and changes in human evolution that created conditions under which a highly pathogenic species of Plasmodium could evolve and be maintained. This scenario supports Anderson and May's (1982) contention that "the coevolutionary trajectory followed by any particular host-parasite association will ultimately depend on the way the virulence and the production of transmission stages of the parasite are linked together: depending on the specifics of this linkage, the coevolutionary course can be towards essentially zero virulence, or to very high vir-

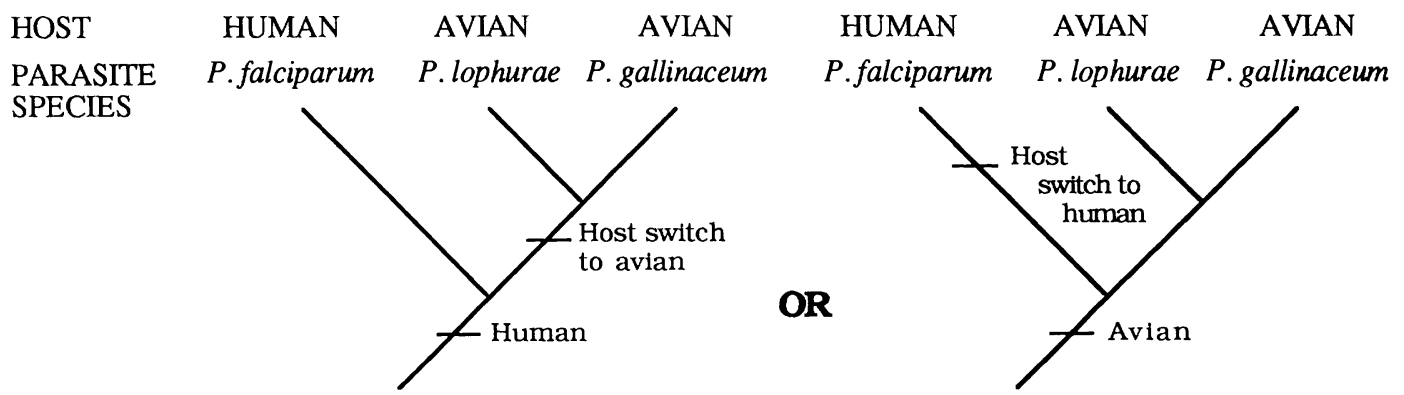

FIGURE 1. Phylogenetic relationships of 3 Plasmodium species (redrawn from Waters et al., 1991). Because humans and birds are not sister taxa, a host switch has occurred within this monophyletic group of parasites. Without information from additional Plasmodium species, however, the direction of that switch cannot be determined because common does not equal primitive. 


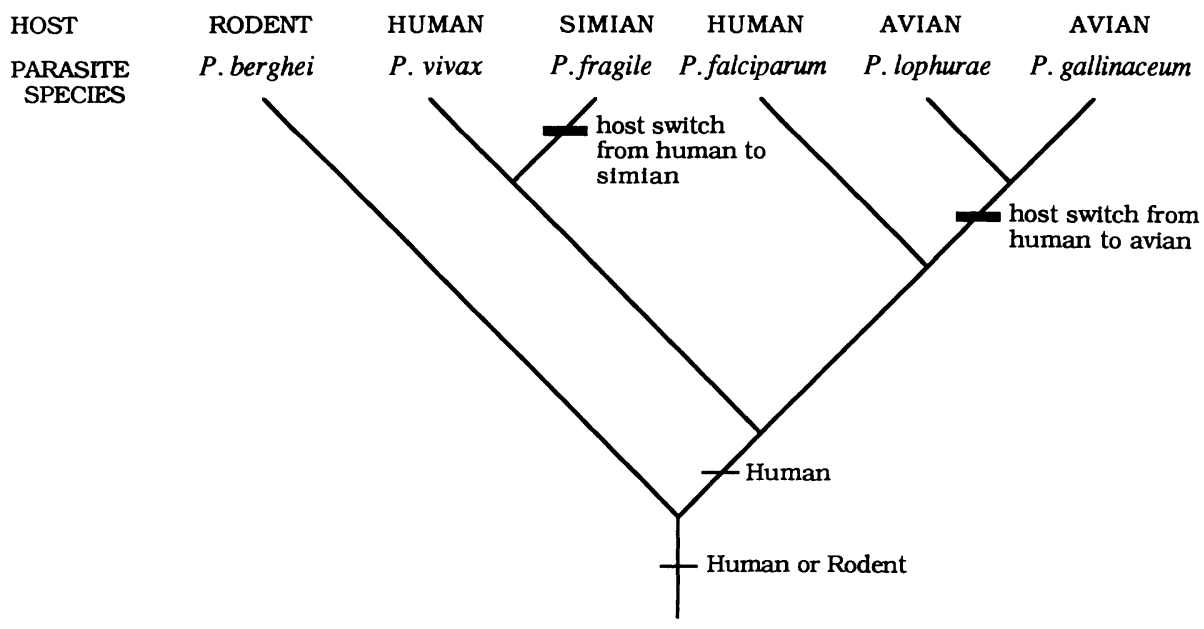

(a)

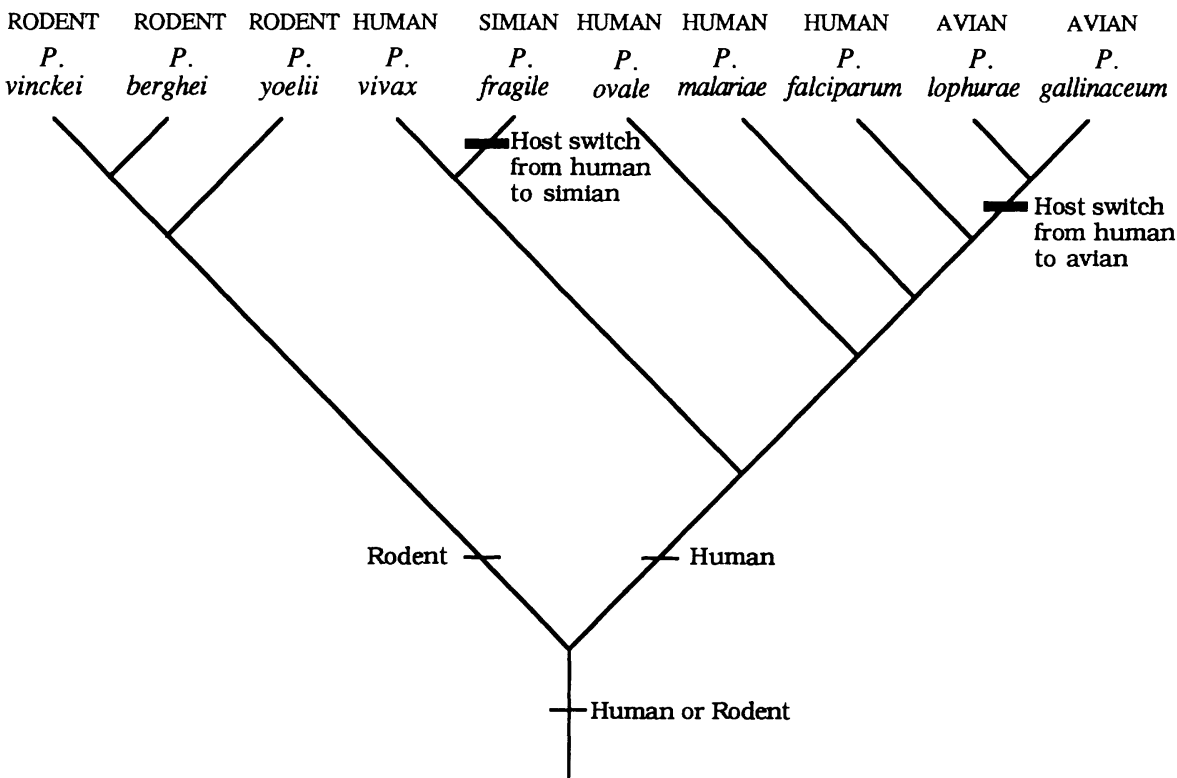

(b)

FIGURE 2. Phylogenetic optimization of vertebrate host types on 2 phylogenetic trees for some species of Plasmodium (redrawn from Waters et al., 1991).

ulence, or to some intermediate grade." These results also indicate that the rise of human agriculture played a role in the evolution of avian malarias. Some avian malarias may have arisen by host switching from humans when agricultural practices brought humans and birds into close contact on a regular basis. If this hypothesis is supported by analysis of further data, then it means that many of the extant avian malarias have evolved over a very short period of time.

The trees produced by Waters et al. (1991) represent a preliminary attempt to address the question of the evolutionary origin of $P$. falciparum. As they noted in their conclusion, a larger number of Plasmodium species needs to be analyzed to provide a more robust 


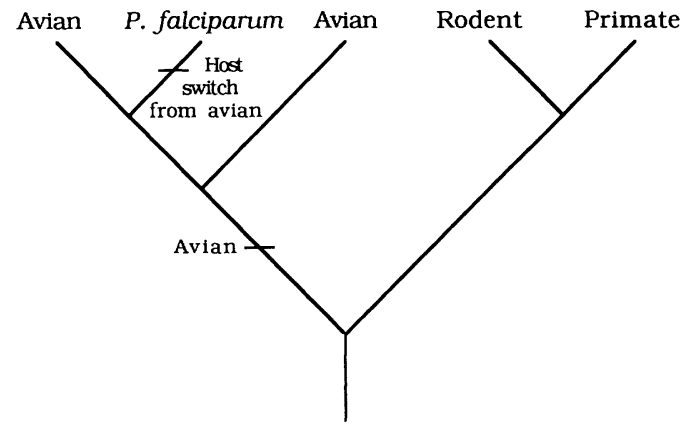

FIGURE 3. Phylogenetic pattern consistent with the hypothesis that Plasmodium falciparum is derived from an ancestor that infected birds. The avian-inhabiting species of Plasmodium plus $P$. falciparum form a clade distinct from the clade of Plasmodium infecting primates.

phylogenetic framework from which to draw conclusions about host and parasite evolutionary patterns. It is possible that the addition of more species will alter the phylogenetic relationships and ultimately provide support for their host switching hypothesis. There are 2 types of phylogenetic patterns that would support the hypothesis that $P$. falciparum is derived from avian Plasmodium spp. In the first case (Fig. 3), the avian Plasmodium species and the primate Plasmodium species, excluding $P$. falciparum, are monophyletic groups (in Fig. 2 the primate-inhabiting Plasmodium species form a paraphyletic group). This would unambiguously support their hypothesis, emphasizing that $P$. falciparum is only distantly related to the other Plasmodium species inhabiting humans. In the second case (Fig. 4), we would explain the origin of $P$. falciparum as the result of a transfer from birds to humans, but the origir of the avian malarias would still involve an earlier host switch from other primate malarias. In this scenario, birds would be said to have acquired Plasmodium from humans, followed by a reverse host switch, resulting in $P$. falciparum. Overall, in order for the Waters et al. (1991) host-switching hypothesis to be supported, $P$. falciparum must be nested within the avian-inhabiting Plasmodium species regardless of the phylogenetic relationships among other Plasmodium species.

In any event, it would appear that a larger number of Plasmodium species needs to be analyzed, and that phylogenetic trees based upon a variety of different data sets need to be generated. This would provide a more powerful phylogenetic framework from which to draw conclusions about host and parasite evolutionary patterns. Comparisons of the new phylogenetic trees with

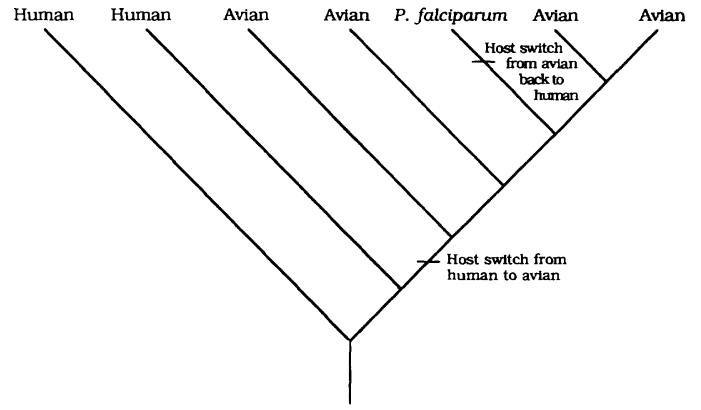

FIgure 4. Phylogenetic pattern consistent with the hypothesis that Plasmodium falciparum is derived from an ancestor that infected birds. The avian species of Plasmodium form a clade nested within primate-infecting Plasmodium species, whereas $P$. falciparum is derived from an ancestor infecting an avian host, thus secondarily infecting primates.

the variety of patterns predicted from various coevolutionary and host-switching phenomena presented herein (and in Ronquist and Nylin [1990] and Brooks and McLennan [1991]) would then provide the most robust explanations about the evolutionary origins of $P$. falciparum. At present, $P$. falciparum remains an enigma.

\section{LITERATURE CITED}

Anderson, R. M., AND R. M. MAY. 1982. Coevolution of hosts and parasites. Parasitology 85: 411426.

Brooks, D. R., And D. A. McLennan. 1991. Phylogeny, ecology and behavior: A research program in comparative biology. University of Chicago Press, Chicago, Illinois, 434 p.

RonQuist, F., AND S. NYlin. 1990. Process and pattern in the evolution of species associations. Systematic Zoology 39: 323-344.

WATERS, A. P., D. G. Higgins, AND T. F. McCutchan. 1991. Plasmodium falciparum appears to have arisen as a result of lateral transfer between avian and human hosts. Proceedings of the National Academy of Sciences USA 88: 3140-3144.

Wiley, E. O., D. Siegel-CaUSey, D. R. BRoOKS, AND V.A. FUNK. 1991. The compleat cladist: A primer of phylogenetic methods. University of Kansas Press, Lawrence, Kansas, 158 p.

Daniel R. Brooks and Deborah A. McLennan, Department of Zoology, University of Toronto, Toronto, Ontario, Canada M5S $1 \mathrm{~A} 1$. 